\title{
Remote System for Detection of Low-Levels of Methane Based on Photonic Crystal Fibres and Wavelength Modulation Spectroscopy
}

\author{
J. P. Carvalho, ${ }^{1,2}$ H. Lehmann, ${ }^{3}$ H. Bartelt, ${ }^{3}$ F. Magalhães, ${ }^{1,4}$ R. Amezcua-Correa, ${ }^{5}$ \\ J. L. Santos, ${ }^{1,2}$ J. Van Roosbroeck, ${ }^{6}$ F. M. Araújo, ${ }^{1}$ L. A. Ferreira, ${ }^{1}$ and J. C. Knight ${ }^{5}$ \\ ${ }^{1}$ INESC Porto, Optoelectronics and Electronic Systems Unit, Rua do Campo Alegre 687, 4169-007 Porto, Portugal \\ ${ }^{2}$ Physics Department, Faculty of Sciences, University of Porto, Rua do Campo Alegre 687, 4169-007 Porto, Portugal \\ ${ }^{3}$ IPHT, Albert Einstein Straße 9, 07745 Jena, Germany \\ ${ }^{4}$ Department of Electrical and Computer Engineering, Faculty of Engineering, University of Porto, Rua Dr. Roberto Frias, \\ $s / n$ 4200-065 Porto, Portugal \\ ${ }^{5}$ Department of Physics, University of Bath, Claverton Down, Bath BA2 7AY, UK \\ ${ }^{6}$ FOSeS, Cipalstraat 14, B-2440 Geel, Belgium
}

Correspondence should be addressed to J. P. Carvalho, joel.carvalho@inescporto.pt

Received 6 March 2009; Accepted 22 June 2009

Recommended by Valerio Pruneri

In this work we described an optical fibre sensing system for detecting low levels of methane. The properties of hollow-core photonic crystal fibres are explored to have a sensing head with favourable characteristics for gas sensing, particularly in what concerns intrinsic readout sensitivity and gas diffusion time in the sensing structure. The sensor interrogation was performed applying the Wavelength Modulation Spectroscopy technique, and a portable measurement unit was developed with performance suitable for remote detection of low levels of methane. This portable system has the capacity to simultaneously interrogate four remote photonic crystal fibre sensing heads.

Copyright ( 2009 J. P. Carvalho et al. This is an open access article distributed under the Creative Commons Attribution License, which permits unrestricted use, distribution, and reproduction in any medium, provided the original work is properly cited.

\section{Introduction}

1.1. Optical Detection of Methane. Methane is an extremely explosive gas and one of the main constituents of natural gas; so its detection is subject of major importance. The idea of sensing methane by laser absorption was first proposed in 1961 by Moore [1] and later demonstrated by Grant [2] in 1986 using an He-Ne laser. Although methane has a strong absorption line at $3.3 \mu \mathrm{m}$, this wavelength region is not suited for optical fibre sensor applications due to the high losses in standard optical fibres and also since it is difficult to fabricate laser diodes operating at wavelengths higher than $2.2 \mu \mathrm{m}$ at room temperature. In order to effectively use the currently available low-loss optical fibres, remote detection in the near infrared around $1.1-1.8 \mu \mathrm{m}$ is desirable, where optical fibres have minimum transmission losses $(<1 \mathrm{~dB} / \mathrm{km})$. Methane has two absorption bands in this region, corresponding to wavelengths of $1.33 \mu \mathrm{m}$ and $1.65 \mu \mathrm{m}$. It was found that the $1.65 \mu \mathrm{m}$ band of methane absorption is more suitable considering the lower loss of the optical fibre in this region, the fact that the absorption coefficients are larger, and also the circumstance that the spectral widths are broader than those in the $1.33 \mu \mathrm{m}$ band [3].

Several authors have proposed many configurations using laser diodes, in particular, distributed feedback (DFB) lasers with almost monochromatic emission, having bandwidths much narrower than the individual gas absorption lines. These devices are a suitable solution for optical-based gas sensing since DFB laser technology has reached maturity due to its broad use in telecommunication systems, with the consequent large reduction of the associated costs.

In 1992, Uehara and Tai [4] demonstrated highsensitivity real time remote detection of methane in air with a DFB operating at $1.65 \mu \mathrm{m}$ (transmission and reflection schemes). Silveira and Grasdepot [5] presented a methane optical sensor using a $1.31 \mu \mathrm{m}$ DFB laser and proposed a 


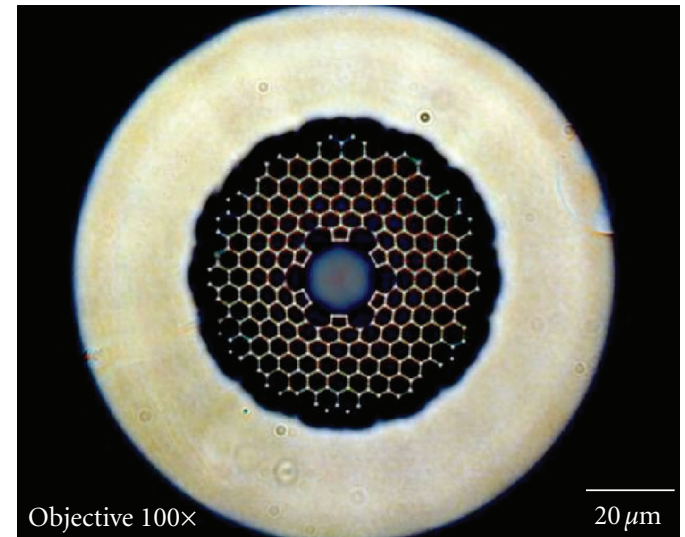

(a)

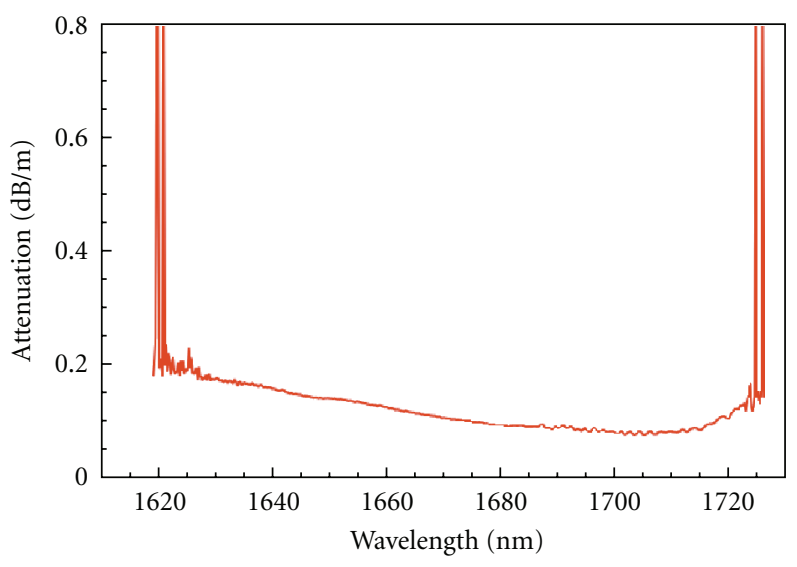

(b)

Figure 1: (a) Optical microscope image of a 7-cell HC-PCF at $1670 \mathrm{~nm}$; (b) spectral fibre transmission.

new type of signal processing technique based on amplitude modulation which provides autocalibration.

The performance limitations of a fibre optic methane sensor using GRIN lenses in either transmission or reflective configurations were examined by Stewart et al. [6]. DFB lasers exhibit very long coherence lengths, and, therefore, multireflections occurring within the gas cells and joints/connectors give rise to interference signals which produce harmonics in the output indistinguishable from the gas signals.

In 2000, Iseki et al. [7] developed a portable remote methane sensor based on frequency modulation using a DFB laser, where a dithering technique is employed, and the first and second derivatives of the absorption line are directly related with the gas concentration.

Chan et al. [8, 9] developed an optical remote sensing system for differential absorption measurement of various inflammable, explosive, and polluting substances employing low-loss optical fibre networks and near-infrared high radiant LED. The highly sensitive technique was achieved employing the power-balanced two-wavelength differential absorption method in the system, which enables direct detection of differential absorption signals for the specific molecule being monitored.

In 2003, Whitenett et al. [10] reported an alternative optical configuration for environmental monitoring applications, namely, the utilization of cavity ringdown spectroscopy using an Erbium Doped Fibre Amplifier (EDFA). This configuration monitors the exponential decay of a light pulse inside a gas chamber that ideally exhibits very high finesse, causing therefore 1/e ring-down time to be very long and very sensitive to small changes in the cavity loss, as induced, for example, by a gas absorber in the cavity.

Photoacoustic spectroscopy (Kosterev et al. [11]) is another technique for detection of absorbing analytes, and it relies on the photoacoustic effect. In this interesting technique, the sample gas is confined in a chamber, where modulated (e.g., chopped) radiation enters via a transparent window and is absorbed by active molecular species. The temperature of the gas thereby increases, leading to a periodic expansion and contraction of the gas volume, synchronous with the modulation frequency of the radiation. This, consequently, produces a pressure wave with amplitude (measured with simple microphones) related to the gas concentration.

Another approach known as multipass transmission absorption spectroscopy can be used and consists of a chamber with mirrors at each end filled with the targeted sample [12]. The beam is folded back and forth through the cell, creating an extended yet defined optical path length in a confined space. Although it presents a high sensitivity, the slow system response to concentration fluctuations and the relatively high volume of the sample required constitute the major disadvantages of this technique.

Other approaches have also been implemented, exploring different types of fibres (e.g., D-fibre) and effects, such as evanescent wave absorption (Culshaw et al. [13]). Their major obstacles, namely, low sensitivity for short interaction lengths, spurious interference effects, and degradation through surface contamination, were analysed (Stewart et al. [14]), and it was determined that the sensitivity of a D-fibre methane gas sensor could be improved by overcoating the flat surface of the fibre with a high index layer, reaching a detection limit lower than 5 ppm (Muhammad et al. [15]).

More recently, several authors proposed new methods for gas detection. Benounis et al. [16] demonstrated a new evanescent fibre sensor based on cryptophane molecules deposited on a PCS (polycarbosilane) fibre. Roy et al. [17] demonstrated a methane sensor based on the utilization of carbon tubes and nanofibres deposited by an electrodeposition technique.

A review of optical sensing methods for methane detection can be found in the work of Magalhães [18].

1.2. Gas Sensing with Photonic Crystal Fibres. The holes in microstructured fibres open up new opportunities for exploiting the interaction of light with gases or liquids. With the possibility of filling the air holes of PCF with gas, with large interaction lengths, new ways to monitor or detect gas are possible. Evanescent field gas sensing in 


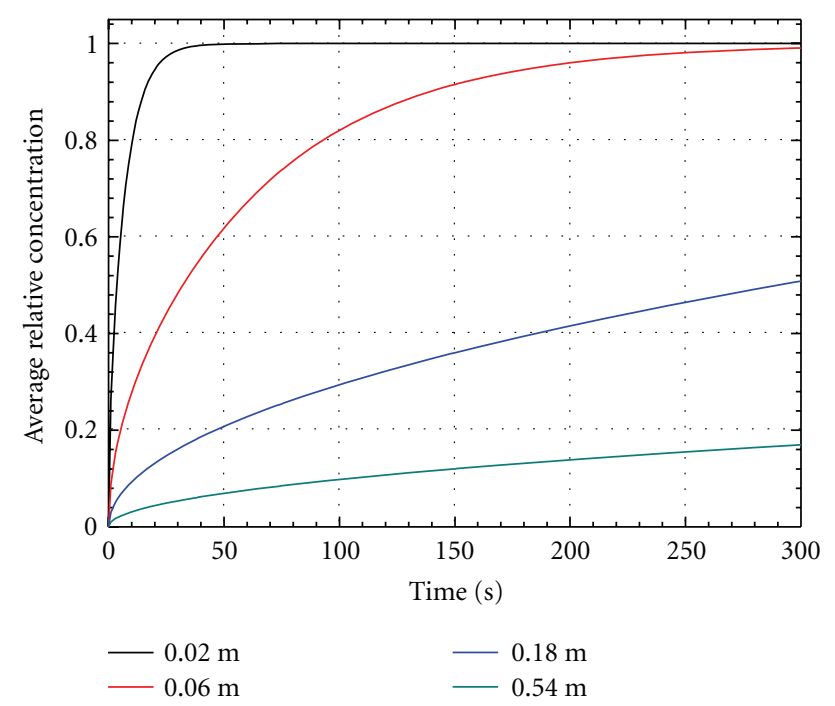

(a)

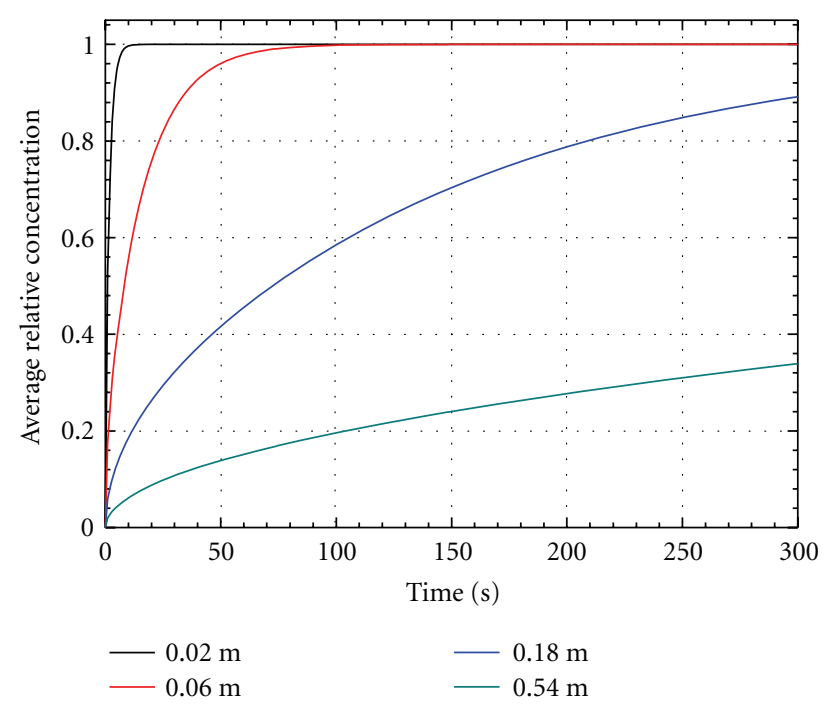

(b)

Figure 2: Time-dependence of the average relative methane concentration inside different lengths of HC-PCF with a single open end (a) and two open ends (b).

the holes of PCF (Monro et al. [19]) or in a fibre random hole structure (Pickrell et al. [20]) has been reported. Hoo et al. [21] demonstrated an absorption spectroscopy evanescent wave acetylene detection system based on a $75 \mathrm{~cm}$ length PCF sensing head. Another work of the same group presents a design and modelling of a PCF-based structure for gas sensing with enhanced characteristics, relying on the amplifying effect associated with the use of the multicoupling gaps (Hoo et al. [22]).

On the other hand, Ritari et al. [23] studied gas characteristics by monitoring the photonic bandgap parameters within hollow-core PCF. Methane detection at $1670 \mathrm{~nm}$ band using hollow-core PCF has been reported (Cubillas et al. [24]). A detection limit of $10 \mathrm{ppm} /$ volume with the system configuration used in the experience is estimated. Cubillas et al. [25] also published a work that describes methane sensing with a hollow-core PCF at $1.3 \mu \mathrm{m}$. Other authors studied the characteristics of gas sensing based on evanescent-wave absorption in solid-core by filling the cladding air holes (Cordeiro et al. [26, 27], Li et al. [28]). Side access to the holes of PCF was demonstrated by Cordeiro et al. [27]. The method consists in inserting the liquid or gas to be sensed laterally to the fibre while the tips are optically monitored. An elegant solution to the problem of the long filling time of the gas inside the fibre was proposed by van Brakel et al. [29], using femtosecond-laser drilling microchannels in both hollow and solid core PCFs.

A comprehensive review of optical sensing based on photonic crystal fibres can be found in the work of Frazão et al. [30].

This work reports the development of an optical sensing system for remote sensitive detection of methane based on hollow-core photonic crystal fibres and Wavelength Modulation Spectroscopy (WMS).

\section{Sensing System}

Hollow-Core Photonic Crystal Fibres (HC-PCF) are structures where the light is guided not by total internal reflection but by a photonic bandgap in the cladding that acts like an insulator for light. The HC-PCF cladding is made with hundreds of periodically spaced air holes in a silica matrix, typically arranged in a honey combed-like pattern. Figure 1(a) shows the cross section of the hollow-core fibre that was used in the experiments. As can be seen, the hollow-core is formed with the suppression of some of such periodically spaced honey combed-like air holes. These fibres exhibit large potential concerning gas sensing since long interaction lengths could be created where light and gas share a common path, therefore, enabling the development of $\mathrm{HC}$ PCF gas sensing heads. This particular fibre is a 7 -cell HCPCF (due to the fact that 7 of those small structures were removed) with a core diameter of $16 \mu \mathrm{m}$. To explore the $1.65 \mu \mathrm{m}$ absorption band of methane, the fibre was designed to have a well defined bandgap in this spectral region. Also, more than $90 \%$ of the light propagates in the core, assuring a high field overlap with the gas and therefore enhanced detection sensitivity [31]. The transmission spectrum of this fibre in the region around $1.65 \mu \mathrm{m}$ is shown in Figure 1(b), obtained using a tuneable laser.

2.1. Diffusion Time. A critical factor in sensing heads projected for detection of dangerous gases is the measurement time. In most cases this is not limited by the intrinsic time constant of the optoelectronic components but by the diffusion time of the gas into the measurement volume. This issue is particularly relevant when these volumes are reduced and accessed only through specific inputs, as is the case when PCF fibres are considered. Therefore, this problem was studied in detail in the context of this work. 


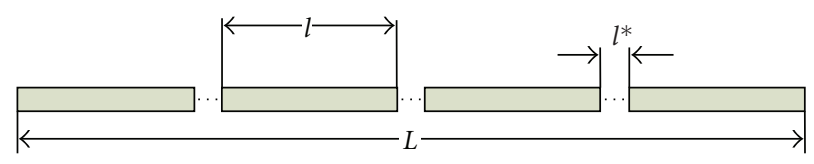

(a)

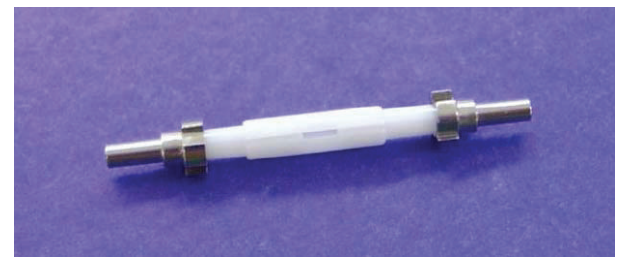

(b)

FIgure 3: (a) Project of the sensing head with periodic openings in the PCF fibre; (b) butt-coupling of HC-PCF pieces using standard zirconium ferrules connected with a standard zirconium mating sleeve.

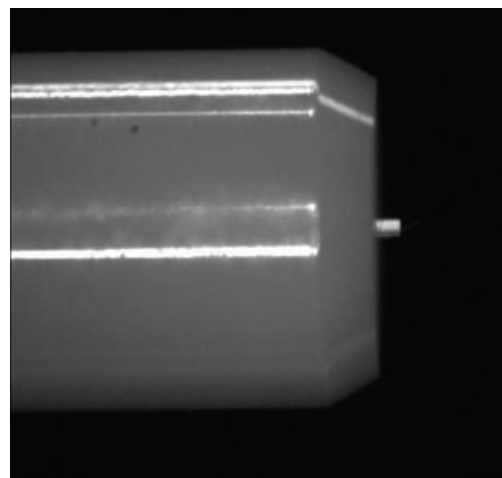

(a)

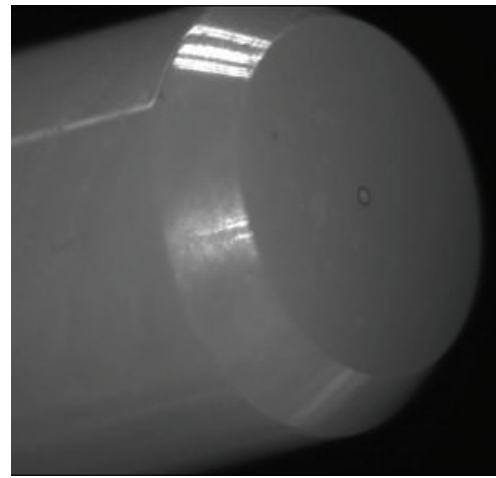

(b)

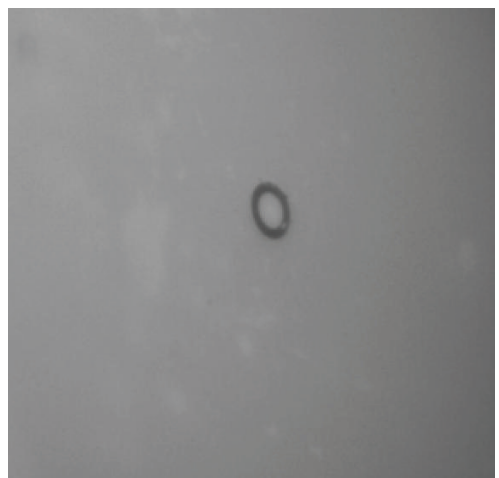

(c)

FIGURe 4: (a) photo of HC-PCF misaligned with the ferrule; (b) photo of the HC-PCF aligned with the ferrule; (c) detailed photo of the HC-PCF aligned with the ferrule.

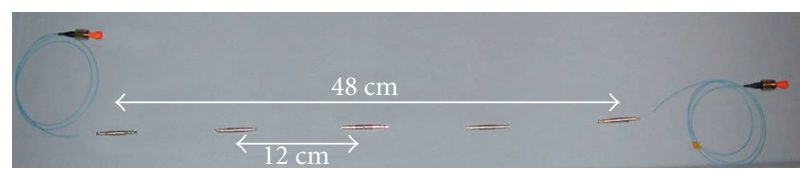

FIGURE 5: Multisegment sensing head containing 4 segments of HCPCF.

There is considered an HC-PCF fibre with one open butt-end immersed in a methane atmosphere and the other butt-end closed. Methane gradually penetrates the fibre by diffusion. The diffusion was characterized by the relative concentration of gas inside the fibre averaged over the fibre length. This concentration can be found by integration of local concentration that is obtained by solving the diffusion equation with corresponding boundary conditions. For the average relative concentration the following expression, represented in a form of an infinite sum, was considered [22]:

$$
C=1-\frac{8}{\pi^{2}} \sum_{j=1,3,5}^{\infty} \frac{1}{j^{2}} \exp \left[-\left(\frac{j \pi}{2 \ell}\right)^{2} D \cdot t\right],
$$

where $\ell$ is the fibre length, $D$ is the diffusion coefficient of methane, and $t$ is the time. The diffusion coefficient for methane in nitrogen is $2.2 \times 10^{-5} \mathrm{~m}^{2} \mathrm{~s}^{-1}$ [32]. Using this value, the dependence of the average relative concentration on time for four fibre lengths $(0.02,0.06,0.18$, and $0.54 \mathrm{~m})$
TABLE 1: Theoretical diffusion times to obtain $90 \%$ average methane concentration in the core of HC-PCF fibres for different lengths.

\begin{tabular}{lcccc}
\hline & \multicolumn{2}{c}{ One open end } & \multicolumn{2}{c}{ Two open ends } \\
$\ell(\mathrm{m})$ & $t(\mathrm{~s})$ & $t(\mathrm{~min})$ & $t(\mathrm{~s})$ & $t(\mathrm{~min})$ \\
\hline 0.02 & 16 & 0.27 & 4 & 0.07 \\
0.06 & 136 & 2.27 & 34 & 0.57 \\
0.18 & 1220 & 20.33 & 305 & 5.08 \\
0.54 & 10980 & 183 & 2745 & 45.75 \\
\hline
\end{tabular}

was plotted. The result is shown in Figure 2(a). For the case of the fibre with two open ends, the average relative methane concentration inside the fibre is obtained from (1) with $2 \ell$ replaced by $\ell$, resulting in the dependence given in Figure 2(b). It should be mentioned that on these calculations surface effects were neglected, given the considerably large diameter of the core of the HC-PCF in comparison to the gas molecules size. Table 1 summarizes the results relative to the diffusion time of methane into the HC-PCF with one and two ends open.

This analysis allows to conclude that the length of the fibre used as the sensing head will directly affect the response time of the sensing system, limiting the time that gases may take to diffuse into the holes. As expected, with two open ends the gas diffusion inside the microstructured fibres happens significantly faster compared with the single open end configuration. 

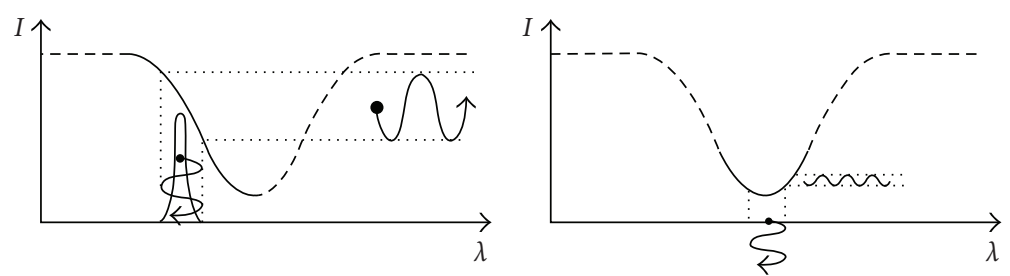

(a)

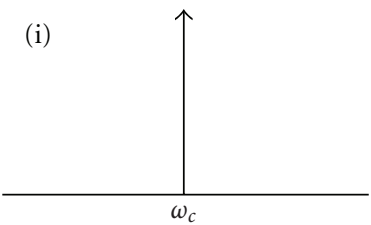

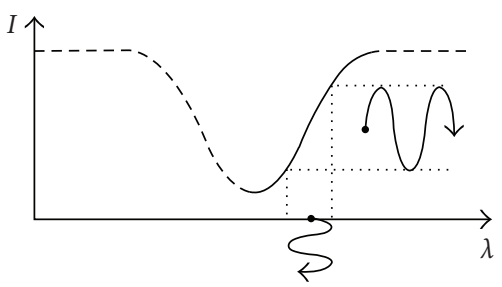

(iii)
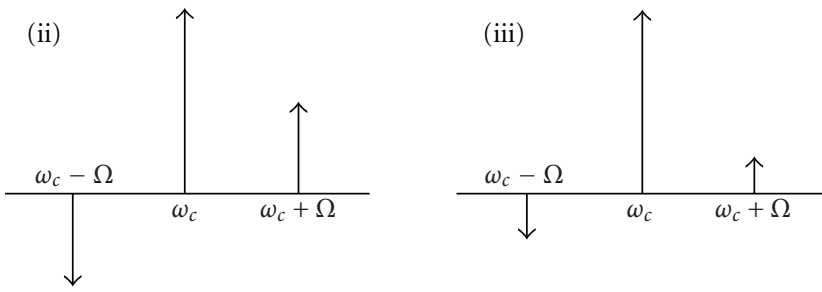

(b)

Figure 6: (a) Wavelength modulation converted to amplitude modulation in Wavelength Modulation Spectroscopy; (b) spectral contents of the optical signal: (i) laser unmodulated; (ii) laser modulated with no absorption; (iii) laser modulated with absorption.

2.2. Sensing Head. To optimize the sensing head sensitivity without compromising the response time a structure was devised where there was considered the introduction of periodic openings along the sensing head fibre, as shown in Figure 3(a). The several hollow-core photonic crystal fibre (HC-PCF) pieces are connected through butt-couplings [31]. As previously stated, the length of each segment can be tuned to control the diffusion speed of the gas inside the sensor and hence the response time of the sensor. In turn, the number of segments determines the total fibre length and, therefore, dictates the sensitivity of the sensing head. Standard zirconium mating sleeves, as shown in Figure 3(b), were found suitable for this butt-coupling approach.

The HC-PCF inside the ferrules was aligned using a nanometre resolution positioning stages as can be seen in Figure 4. The diameter of the sleeves is deliberately slightly smaller than the diameter of the ferrules. A slit along the length of the sleeve gives some extra flexibility to the inner diameter and allows the sleeve to act like a small spring (in the radial direction). This mechanism ensures that the ferrules are clamped inside the sleeve, thus optimizing the alignment. Since this is the standard technology for aligning fibre connectors, the technology is well established, and the components are relatively cheap. Furthermore, the slit in the sleeves is also well suited to allow gas in-diffusion through open gaps. Figure 5 shows a chain of different HC-PCF segments connected with this method. This multisegment sensor was placed inside a silica tube that acts like a gas chamber which facilitates greatly the experimental test. The insertion loss of the sensing head is a consequence of the loss induced by each butt joint and can be quantified as presented in a previous work (Carvalho et al. [31]), where there was characterised the optical coupling in a 19-cell HC-PCF with a core diameter, and thus a guided mode behaviour, quite similar to the 7-cell HC-PCF fibre used here. Typically, it was found that the loss in each butt-joint is smaller than $1 \mathrm{~dB}$.

With adequate sensing head packaging, that involves fibre wrapping of several butt-joints segments, which is possible due to the geometrical flexibility of the optical fibre, the volume increase when the number of butt-joint segments increases can follow a slow grow.

2.3. Interrogation Technique. The ability to have sensitive detection of methane with the previously described sensing head requires the consideration of a high-performance interrogation approach. Therefore, the technique Wavelength Modulated Spectroscopy (WMS) was selected in view of its favourable characteristics (Silver [33]). In WMS, the source wavelength is slowly modulated, sweeping the entire absorption peak, and a higher frequency signal (dithering) is superimposed on this signal. As the emission source wavelength slowly scans through the gas absorption line, the wavelength modulation becomes an amplitude modulation, presenting its highest amplitude as it passes in the highest slope points of the absorption peak, as presented in Figure 6(a). As the schemes in this figure indicate, this interrogation method requires that the optical source should have a linewidth significantly smaller than the absorption line of the gas species to be monitored. Considering the detection bandwidth is shifted to higher frequencies where the laser intensity noise is reduced towards the shot noise, and the signal-to-noise ratio is substantially increased, which means better measurement resolution. This concept is similar to that of data encoding in the side bands of a radio transmission carrier wave. Figure 6(b) shows the spectral output of a frequency modulated laser, where there can be seen the carrier frequency $\omega_{c}$ and the side-band frequencies $\omega_{\mathrm{c}} \pm \Omega$. Therefore, when the laser slowly scans through the 


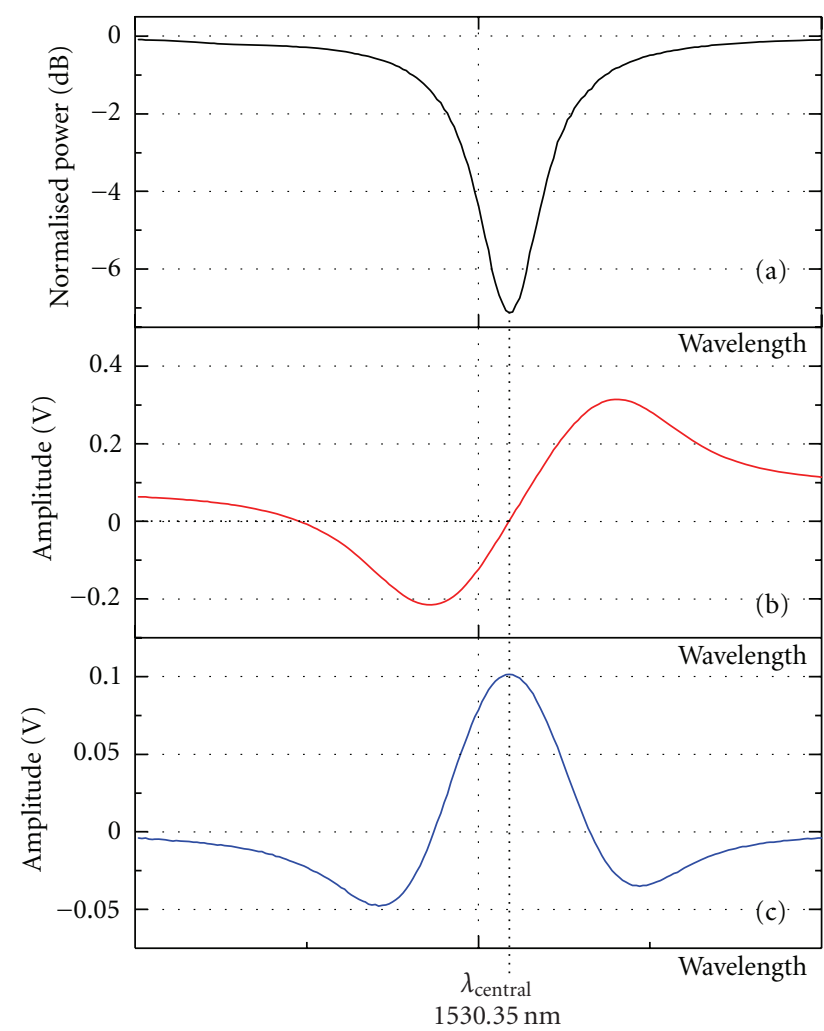

— Acetylene gas cell absorption peak
— 1st harmonic
- 2nd harmonic

FigURE 7: Absorption line of an acetylene gas cell (a) and amplitude of the output signals at the dithering frequency (b) and at double of this frequency (c).

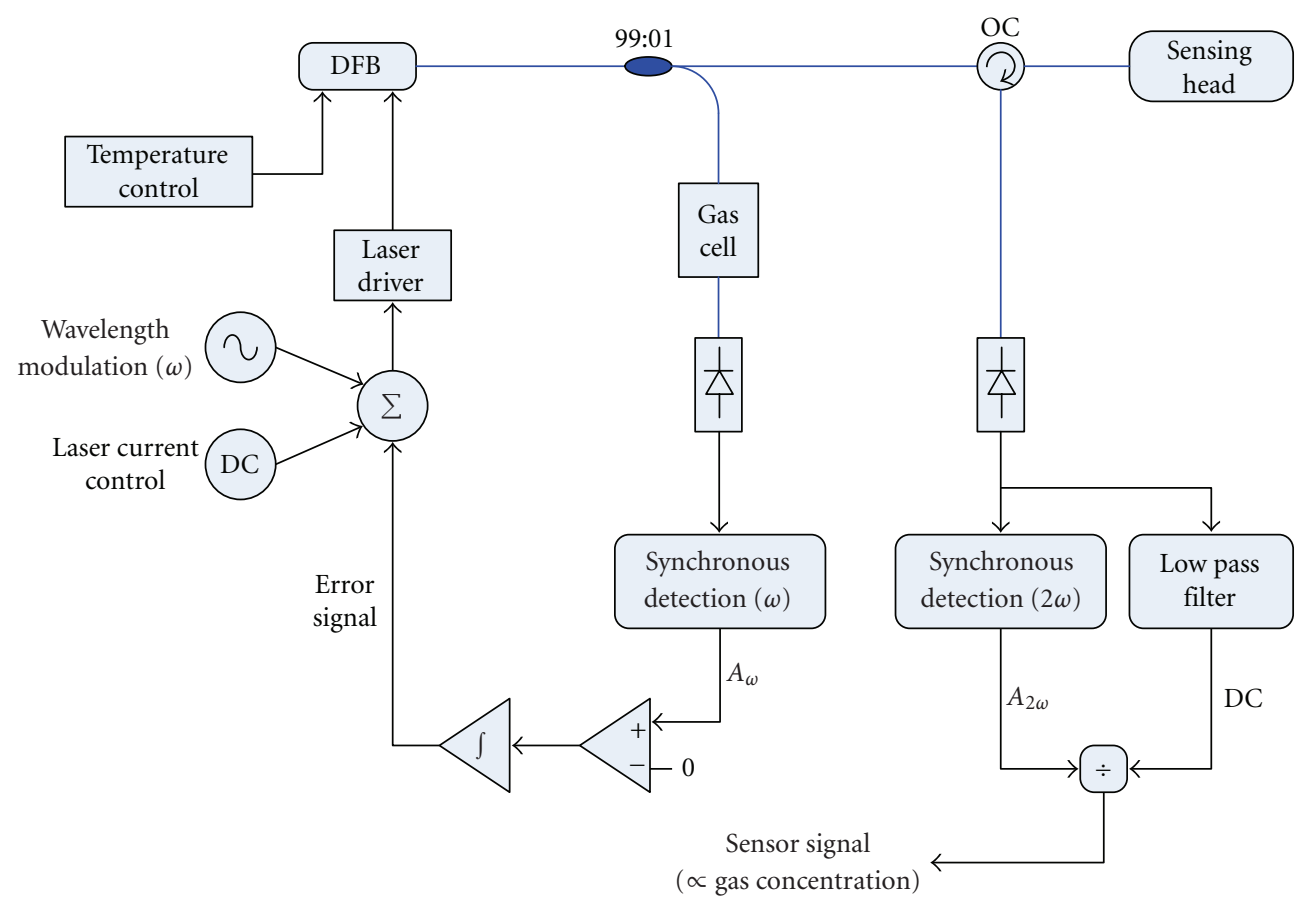

FIGURE 8: Layout of the Sensing head interrogation based on Wavelength Modulated Spectroscopy. 
absorption line, the amount of light absorbed, which by the Lambert-Beer Law is proportional to the gas concentration, is "written" into the side bands. Schematically, this is represented in Figure 6(b) as a decrease in the amplitude of the side bands. Consequently, the absorption information can be retrieved by means of a lock-in amplifier, where a voltage output proportional to gas concentration can be generated.

Some features of this interrogation technique can be better appreciated observing the curves shown in Figure 7. They are relative to the acetylene, the gas that was used in the system development phase because it shows an absorption band around $1530 \mathrm{~nm}$ (Figure $7(\mathrm{a})$; the acetylene strongest absorption peak in the $P$ branch of the $\nu_{1}+\nu_{3}$ band), a highly convenient spectral position considering the large range of optical fibre components that can be used in view of its location in the third optical communications spectral window. The interaction between the acetylene (pressure $\approx 200$ Torr, $\approx 100 \%$ concentration) and the optical field happened in a $U$-bench with $30 \mathrm{~mm}$ length. The laser source used for these experiments was an Avanex-1905 LMI DFB laser optimized for operation at $1530 \mathrm{~nm}$. With the lockin amplifier locked at the dithering frequency, the output signal is the first derivative of the gas absorption line, and it equals zero when the source wavelength is centred in the absorption peak (Figure 7(b)). The lock-in amplifier output for the second harmonic is the derivative trace of the output at the dithering frequency and reaches a maximum at the peak absorption wavelength (Figure 7(c)). Disabling the slow modulation and stabilizing the source emission wavelength at the absorption peak, the dithering gives rise to a transmitted signal with a frequency that is twice the dithering one and with amplitude that depends on the gas concentration.

This detection method thus converts a frequency modulation into an amplitude modulation. The measured signal will contain both AC and DC components. Fluctuations of the optical power (from the source, fibre bends, etc.) will commonly modify the AC and DC components of the signal; so the ratio of the AC component with the $\mathrm{DC}$ component remains fairly unaltered, therefore only affected by the gas concentration. This insensitivity to optical power fluctuations is one of the main advantages of the WMS method.

Figure 8 shows the detailed scheme of the optoelectronic detection technique based in WMS. Ideally, an optical source suited for the WMS method shall have the following properties: high power (assures good signal-to-noise ratios and allows sensor multiplexing), narrow line width (in comparison with the line width of the methane absorption lines), which is also important to enhance the signal-tonoise ratio and tuneable emission wavelength. Distributed Feedback Lasers meet all these demands at affordable cost. A DFB diode can be tuned in wavelength by changing either the temperature or the operating current. While current-tuning is favourable for rapid modulation tasks, thermal tuning has the advantage of providing extremely large mode-hop free tuning ranges. In the configuration presented in the figure, the wavelength of the DFB optical source is modulated with a frequency $\omega$ by means of current tuning. The light is then guided through a directional coupler. Part of the light is sent to a reference gas cell whereas the remaining portion of light is sent to a sensing head. The reference cell is used to keep the laser wavelength locked to the gas absorption line. Monitoring of the light from the reference cell happens with a lock-in amplifier working at the modulation frequency, and the resulting signal is feedback to the laser driver. The light coming from the sensor is analyzed for two components. The DC component is filtered out using a lowpass filter, and the doubled frequency amplitude modulated signal is isolated using synchronous detection at $2 \omega$. The information for gas concentration is obtained from the ratio $S=A_{2 \omega} / D C$, where $A_{2 \omega}$ is the amplitude of the signal synchronously detected at $2 \omega$ and $D C$ the continuous level. This relation yields the absorbance and hence the gas concentration. The resulting signal, as previously stated, should be independent of optical power fluctuations.

In order to enable the variation of gas concentrations and to test the implemented setup with the HC-PCF-based sensing heads, a gas chamber was developed (Figure 9). The chamber is hermetically sealed and has one gas input and output as well as a pressure manometer and an optical feedthrough to allow the entrance of fibres. Inside of this chamber there are also two U-benches with different path lengths (30 $\mathrm{mm}$ and $6.8 \mathrm{~mm}$ ).

A LabVIEW application has been developed to control all the signal generation, acquisition as well as all the single processing stages. With a standard computer and a DAQ board a virtual instrumentation workbench has been implemented, with the objective to perform system integration of a portable and customizable system for remote detection of gas species

\section{Results}

The experimental arrangement implemented to test the gas diffusion time into the HC-PCF is shown in Figure 10(a). Standard SMF transmitted light to the HC-PCF and guided it to the detection unit. After injection of gas into the chamber (a mixture of $5 \%$ of methane and $95 \%$ of nitrogen), the decay of transmitted light with time caused by the absorbance of the gas inside the HC-PCF was registered. The length of the sensing head HC-PCF was $13.7 \mathrm{~cm}$. The optical source, a DFB laser optimized to work at $1666 \mathrm{~nm}$, was emitting at the strongest methane absorption line in the $Q$ branch of the $2 v_{3}$ band (Rothman et al. [34]). The frequency of the dithering signal that modulates the DFB was $500 \mathrm{~Hz}$. To allow gas diffusion, the gap between the input and output SMF and the HC-PCF was guaranteed by the use of angled ferrules (FC/APC) in the side of the standard fibres (Figure 10(b); $x \approx 176 \mu \mathrm{m})$. These ferrules, exhibiting an angle of $8^{\circ}$, were chosen because they do not permit Fresnel (silica-air interface) back reflections to be guided.

The obtained results for methane diffusion inside the HC-PCF are presented in Figure 11. It can be observed that the time taken to achieve $95 \%$ of the steady state was about 248 seconds, while the theoretically predicted value (obtained from ( 1 ) with $2 \ell$ replaced by $\ell$ ) was about 


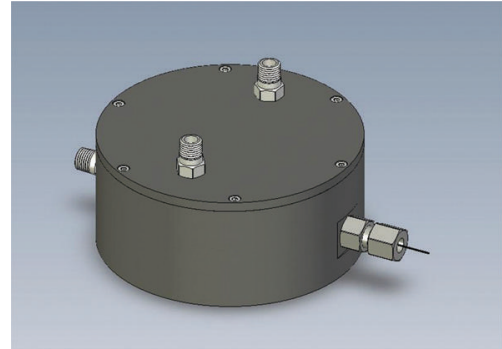

(a)

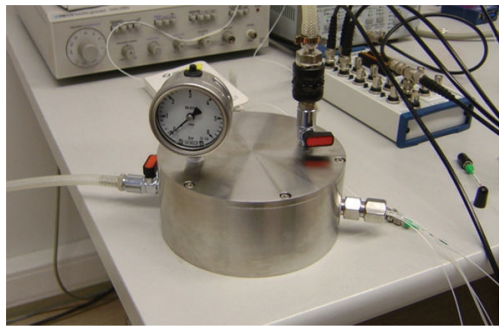

(c)

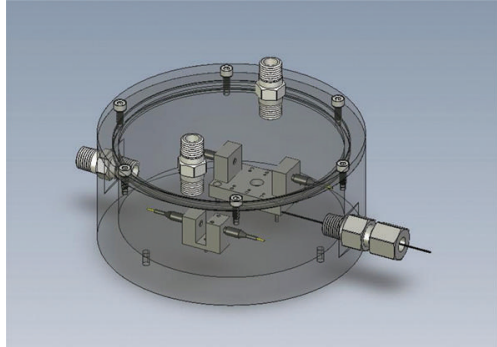

(b)

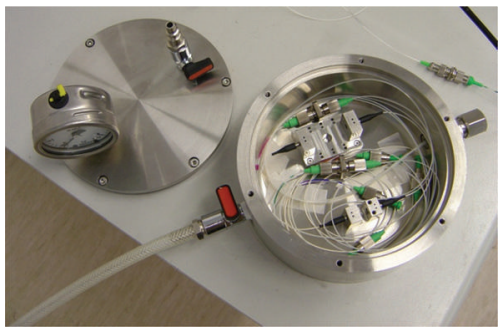

(d)

Figure 9: Gas chamber for test of the sensing system: (a) and (b) computer generated pictures; (c) and (d) photographs of the implemented gas chamber (the U-benches shown in (d) were used to test the WMS interrogation technique).

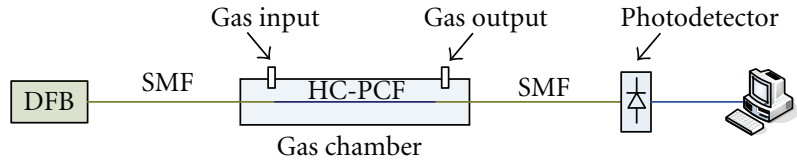

(a)

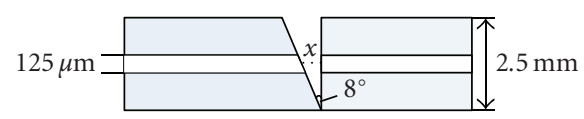

(b)

Figure 10: (a) Setup used to measure the diffusion time of gas inside the HC-PCF based sensing head; (b) illustration of the joint between SMF and HC-PCF.

241 seconds, thus leading to a relative error of $\sim 2.8 \%$. Therefore, this agreement confirms the reliability of the adopted model.

The detection limit of the methane sensing head can be estimated from the signal-to-noise ratio (SNR) of the demodulated signal. The second harmonic peak at $1000 \mathrm{~Hz}$ shows a SNR of $\approx 50 \mathrm{~dB}$, and it corresponds to a methane concentration of $5 \%$, or $50000 \mathrm{ppm}$. In a linear scale, the $\mathrm{SNR}$ is $\approx 316$, which means that we are able to detect a concentration smaller by this factor. Therefore, the sensing head with $13.7 \mathrm{~cm}$ length and opened in both sides permits a methane detection limit of $\approx 158 \mathrm{ppm}$.

If more segments are considered in the sensing head, as shown in Figure 5, the detection limit shall improve proportionally to the increase of the light interaction length with the gas. However, there was noticed the presence of a deleterious effect associated with superficial modes at the

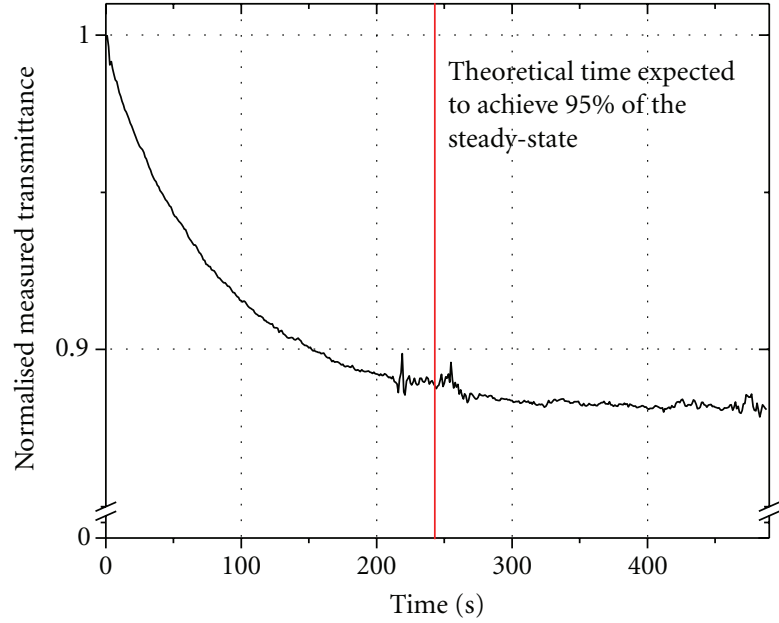

Figure 11: (Experimental results for the diffusion time of 5\% of $\mathrm{CH}_{4}$ inside an HC-PCF with a length of $13.7 \mathrm{~cm}$ (two open ends). The theoretical expected value ( 241 seconds) is shown by the vertical line, while the experimental one is 248 seconds.

boundary of the fibre hollow core. These modes induce interference effects that reduce the signal-to-noise ratio. Research is going on aiming to overcome this problem.

Looking for field application of the R\&D above described, an optoelectronic portable measurement unit was developed to measure gas concentration with HC-PCFbased sensors. The portable unit integrates a computer (motherboard, memory, hard-disk, touch-screen, etc.), an optoelectronic board and optical switching capability for multiplexing of four remote photonic crystal fibre sensing 


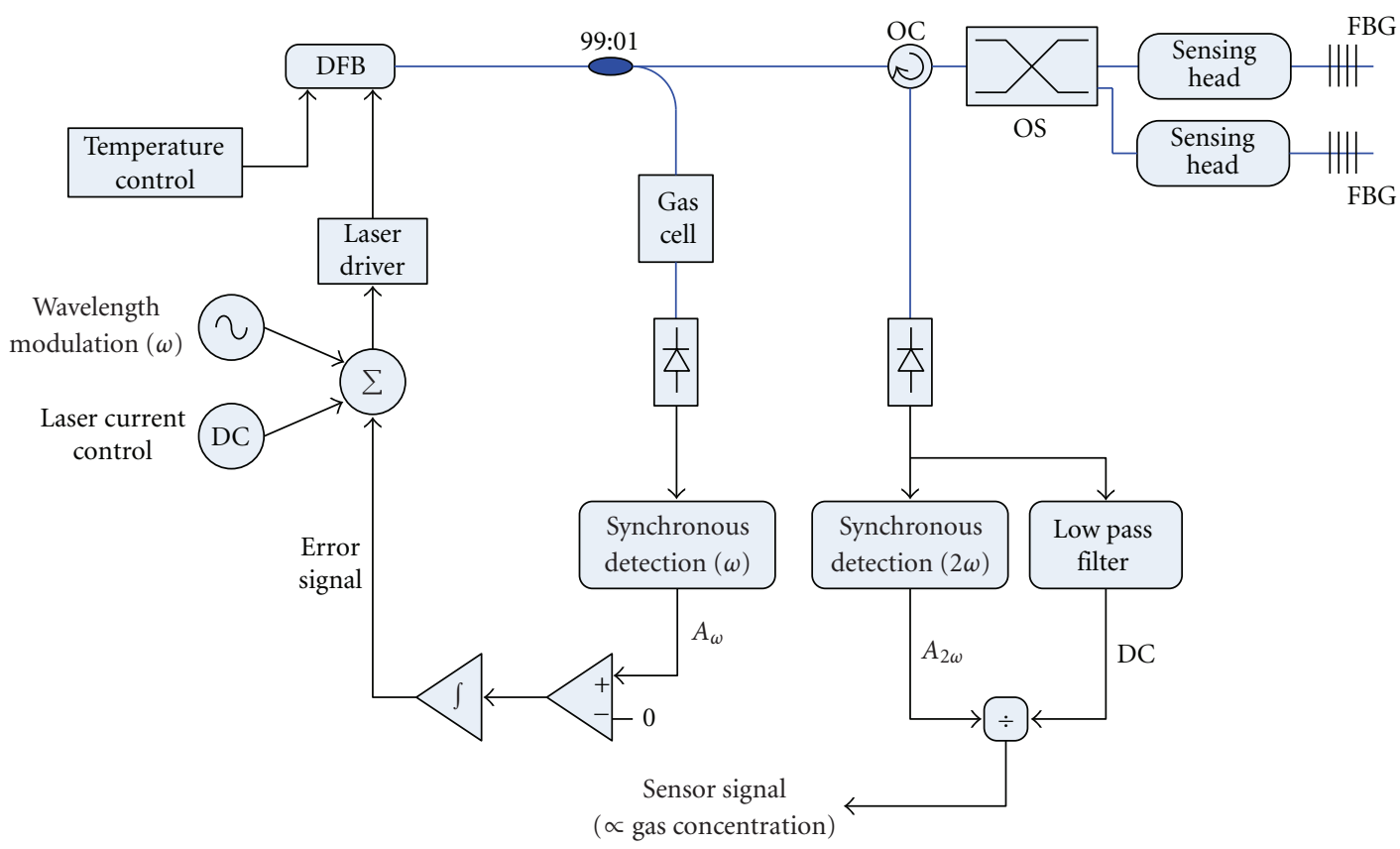

(a)

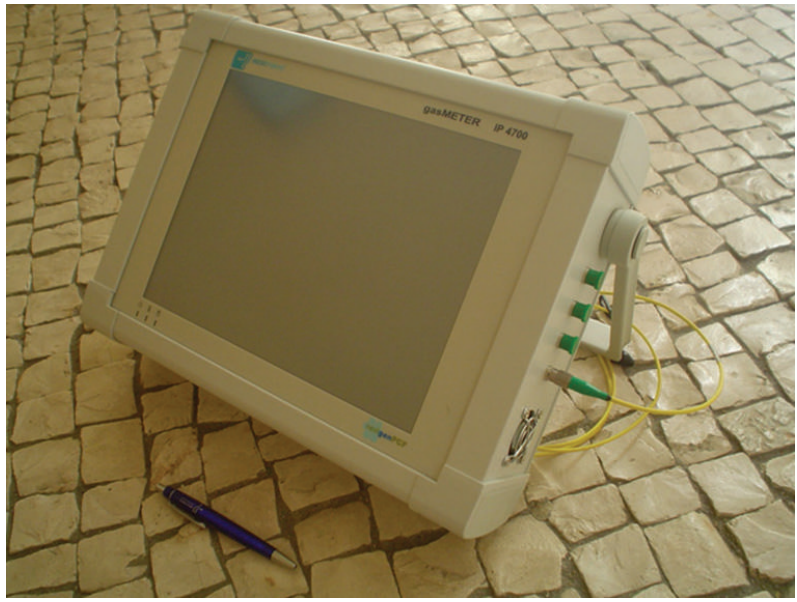

(b)

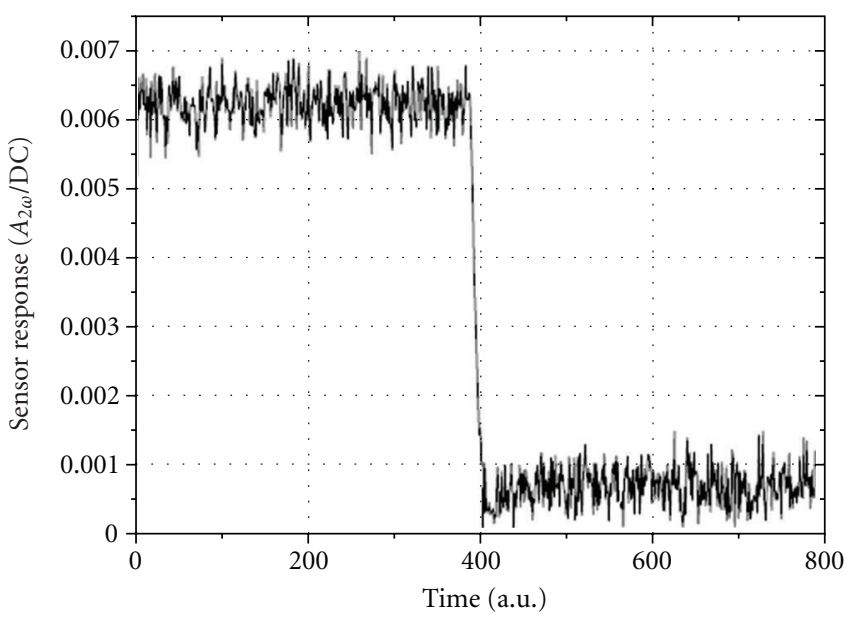

(c)

FIGURE 12: (a) Diagram of the gas measurement unit prototype; (b) photo of the unit; (c) response for a methane concentration change.

heads. A LabVIEW application was developed to control the measurement unit system which presents a graphical interface to the user. A diagram of the implemented system is presented in Figure 12(a), while Figure 12(b) shows a photo of the equipment. Figure 12(c) gives a unit screen plot representing the system response to a change of methane concentration in the sensing head.

\section{Conclusion}

This work reported the development of an optical fibre sensing system for detection of low levels of methane, based on a sensing concept that explores the favourable characteristics for gas sensing of hollow-core photonic crystal fibres together with the Wavelength Modulation Spectroscopy interrogation technique. A sensing head structure was designed that is compatible with sensitive methane detection and acceptable measurement time, which is related with the gas diffusion time into the measurement volume. The results obtained confirm the potential of this sensing approach, and aiming field applications a portable measurement unit was developed that has the capacity to simultaneously interrogate four remote photonic crystal fibre sensing heads.

\section{References}

[1] C. B. Moore, "Gas-laser frequency selection by molecular absorption," Applied Optics, vol. 4, no. 2, pp. 252-253, 1965. 
[2] W. B. Grant, "He-Ne and $\mathrm{Cw}$ Co2-laser long-path systems for gas-detection," Applied Optics, vol. 25, no. 5, pp. 709-719, 1986.

[3] B. Culshaw, G. Stewart, F. Dong, C. Tandy, and D. Moodie, "Fibre optic techniques for remote spectroscopic methane detection-from concept to system realisation," Sensors and Actuators B, vol. 51, no. 1-3, pp. 25-37, 1998.

[4] K. Uehara and H. Tai, "Remote detection of methane with a 1.66- $\mu \mathrm{u}-\mathrm{M}$ diode-laser," Applied Optics, vol. 31, no. 6, pp. 809$814,1992$.

[5] J. P. Silveira and F. Grasdepot, " $\mathrm{CH}_{4}$ optical sensor using a $1.31 \mu \mathrm{m}$ DFB laser diode," Sensors and Actuators B, vol. 25, no. 1-3, pp. 603-606, 1995.

[6] G. Stewart, A. Mencaglia, W. Philp, and W. Jin, "Interferometric signals in fiber optic methane sensors with wavelength modulation of the DFB laser source," Journal of Lightwave Technology, vol. 16, no. 1, pp. 43-53, 1998.

[7] T. Iseki, T. Hideo, and K. Kimura, "A portable remote methane sensor using a tunable diode laser," Measurement Science \& Technology, vol. 11, no. 6, pp. 594-602, 2000.

[8] K. Chan, H. Ito, and H. Inaba, "Remote-sensing system for near-infrared differential absorption of $\mathrm{CH}_{4}$ gas-using lowloss optical fiber link," Applied Optics, vol. 23, no. 19, pp. 34153420, 1984.

[9] K. Chan, H. Ito, H. Inaba, and T. Furuya, "10 km-long fibreoptic remote sensing of $\mathrm{CH}_{4}$ gas by near infrared absorption," Applied Physics B, vol. 38, no. 1, pp. 11-15, 1985.

[10] G. Whitenett, G. Stewart, K. Atherton, B. Culshaw, and W. Johnstone, "Optical fibre instrumentation for environmental monitoring applications," Journal of Optics A, vol. 5, no. 5, pp. S140-S145, 2003.

[11] A. A. Kosterev, Y. A. Bakhirkin, and F. K. Tittel, "Ultrasensitive gas detection by quartz-enhanced photoacoustic spectroscopy in the fundamental molecular absorption bands region," Applied Physics B, vol. 80, no. 1, pp. 133-138, 2005.

[12] "State-of-the-Art laser-based gas sensing," in Photonics Spectra, Laurin Publishing, 2006.

[13] B. Culshaw, F. Muhammad, G. Stewart, et al., "Evanescent wave methane detection using optical fibres," Electronics Letters, vol. 28, no. 24, pp. 2232-2234, 1992.

[14] G. Stewart, W. Jin, and B. Culshaw, "Prospects for fibre-optic evanescent-field gas sensors using absorption in the nearinfrared," Sensors and Actuators B, vol. 38, no. 1-3, pp. 42-47, 1997.

[15] F. A. Muhammad, G. Stewart, and W. Jin, "Sensitivity enhancement of D-fiber methane gas sensor using high-index overlay," Power Engineering Journal, vol. 7, no. 3, pp. 115-118, 1993.

[16] M. Benounis, N. Jaffrezic-Renault, J.-P. Dutasta, K. Cherif, and A. Abdelghani, "Study of a new evanescent wave optical fibre sensor for methane detection based on cryptophane molecules," Sensors and Actuators B, vol. 107, no. 1, pp. 32-39, 2005.

[17] R. K. Roy, M. P. Chowdhury, and A. K. Pal, "Room temperature sensor based on carbon nanotubes and nanofibres for methane detection," Vacuum, vol. 77, no. 3, pp. 223-229, 2005.

[18] F. Magalhães, Development of gas detection systems based on microstructured optical fibres, MSc thesis, DEEC/FEUPElectrical and Computers Engineering, Department of the Faculty of Engineering, University of Porto, Porto, Portugal, 2008.

[19] T. M. Monro, D. J. Richardson, and P. J. Bennett, "Developing holey fibres for evanescent field devices," Electronics Letters, vol. 35, no. 14, pp. 1188-1189, 1999.
[20] G. Pickrell, W. Peng, and A. Wang, "Random-hole optical fiber evanescent-wave gas sensing," Optics Letters, vol. 29, no. 13, pp. 1476-1478, 2004.

[21] Y. L. Hoo, W. Jin, H. L. Ho, D. N. Wang, and R. S. Windeler, "Evanescent-wave gas sensing using microstructure fiber," Optical Engineering, vol. 41, no. 1, pp. 8-9, 2002.

[22] Y. L. Hoo, W. Jin, C. Shi, H. L. Ho, D. N. Wang, and S. C. Ruan, "Design and modeling of a photonic crystal fiber gas sensor," Applied Optics, vol. 42, no. 18, pp. 3509-3515, 2003.

[23] T. Ritari, J. Tuominen, H. Ludvigsen, et al., "Gas sensing using air-guiding photonic bandgap fibers," Optics Express, vol. 12, no. 17 , pp. 4080-4087, 2004.

[24] A. M. Cubillas, M. Silva-Lopez, J. M. Lazaro, O. M. Conde, M. N. Petrovich, and J. M. Lopez-Higuera, "Methane detection at 1670-nm band using a hollow-core photonic bandgap fiber and a multiline algorithm," Optics Express, vol. 15, no. 26, pp. 17570-17576, 2007.

[25] A. M. Cubillas, J. M. Lazaro, M. Silva-Lopez, O. M. Conde, M. N. Petrovich, and J. M. Lopez-Higuera, "Methane sensing at $1300 \mathrm{~nm}$ band with hollow-core photonic bandgap fibre as gas cell," Electronics Letters, vol. 44, no. 6, pp. 403-405, 2008.

[26] C. M. B. Cordeiro, E. M. dos Santos, C. H. B. Cruz, C. J. S. de Matos, and D. S. Ferreira, "Lateral access to the holes of photonic crystal fibers - selective filling and sensing applications," Optics Express, vol. 14, no. 18, pp. 8403-8412, 2006.

[27] C. M. B. Cordeiro, M. A. R. Franco, G. Chesini, et al., "Microstructured-core optical fibre for evanescent sensing applications," Optics Express, vol. 14, no. 26, pp. 13056-13066, 2006.

[28] S.-G. Li, S.-Y. Liu, Z.-Y. Song, et al., "Study of the sensitivity of gas sensing by use of index-guiding photonic crystal fibers," Applied Optics, vol. 46, no. 22, pp. 5183-5188, 2007.

[29] A. van Brakel, C. Grivas, M. N. Petrovich, and D. J. Richardson, "Micro-channels machined in microstructured optical fibers by femtosecond laser," Optics Express, vol. 15, no. 14, pp. 8731-8736, 2007.

[30] O. Frazão, J. L. Santos, F. Araújo, and L. A. Ferreira, "Optical sensing with photonic crystal fibres," Laser \& Photonic Reviews, vol. 2, no. 6, pp. 449-459, 2008.

[31] J. P. Carvalho, F. Magalhães, O. V. Ivanov, et al., "Evaluation of coupling losses in hollow-core photonic crystal fibres," in Proceedings of the 3rd European Workshop on Optical Fibre Sensors, vol. 6619, 2007.

[32] D. R. Lide, CRC Handbook of Chemistry and Physics, CRC Press, Boca Raton, Fla, USA, 1997.

[33] J. A. Silver, "Frequency-modulation spectroscopy for trace species detection: theory and comparison among experimental methods," Applied Optics, vol. 31, no. 6, pp. 707-717, 1992.

[34] L. S. Rothman, D. Jacquemart, A. Barbe, et al., "The HITRAN 2004 molecular spectroscopic database," Journal of Quantitative Spectroscopy and Radiative Transfer, vol. 96, no. 2, pp. 139204, 2005. 

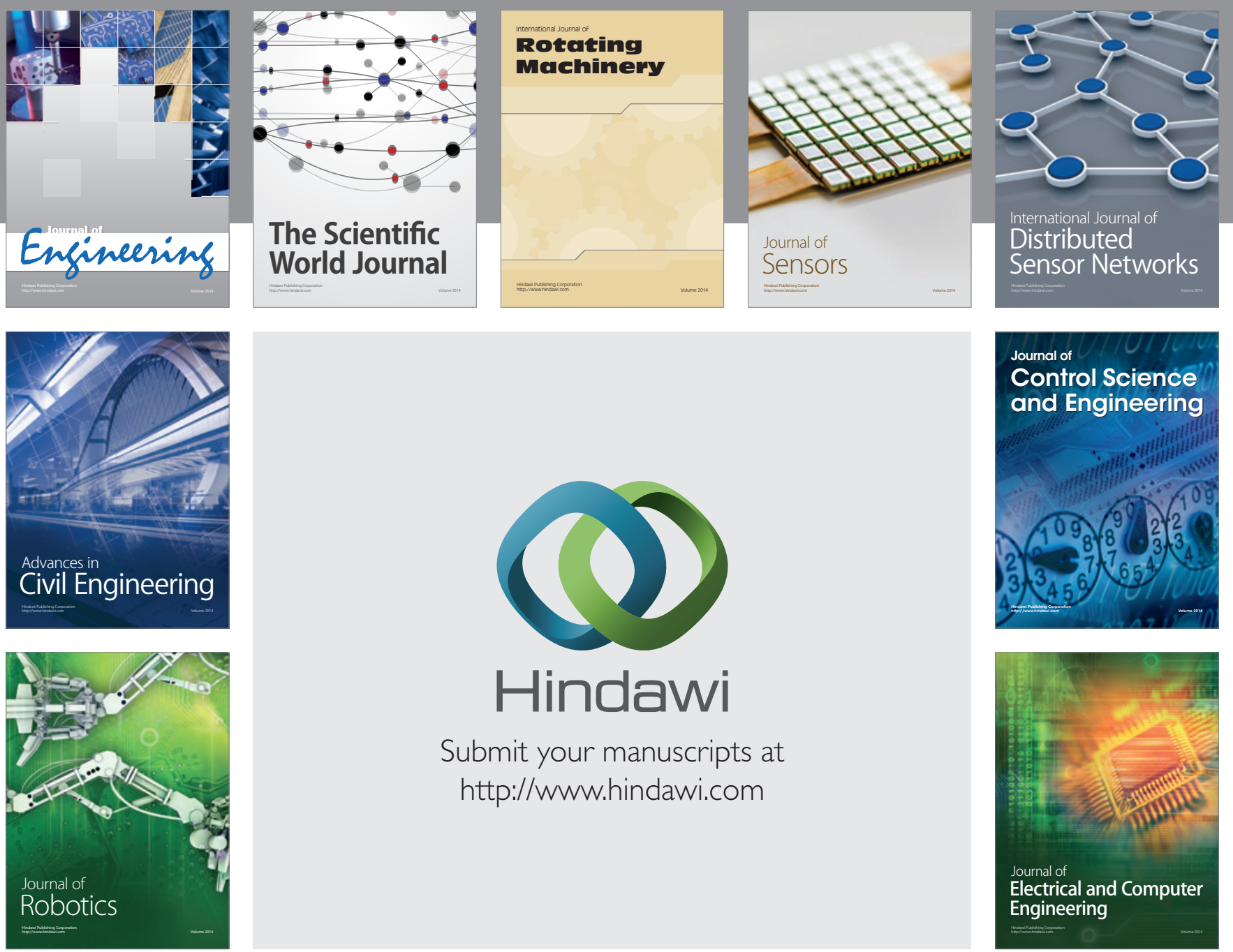

Submit your manuscripts at

http://www.hindawi.com
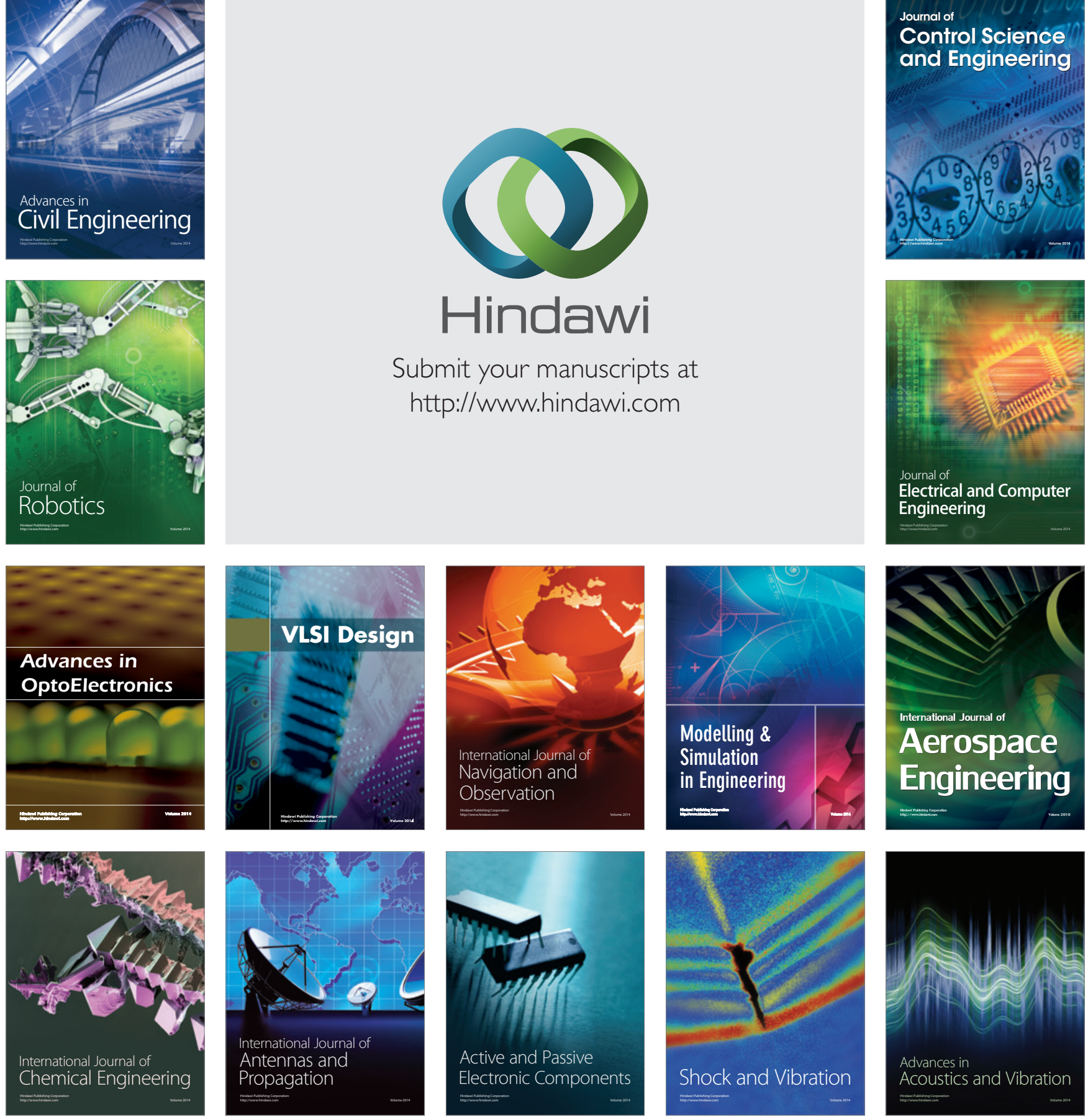